\title{
Interpretation of the mechanism associated with turbulent drag reduction in terms of anisotropy invariants
}

\author{
B. FROHNAPFEL ${ }^{1}$, P. LAMMERS ${ }^{2}$, J. JOVANOVIĆ \\ AND F. DURS T ${ }^{1}$ \\ ${ }^{1}$ Institute of Fluid Mechanics, Friedrich-Alexander University Erlangen-Nuremberg, \\ Cauerstr. 4, 91058 Erlangen, Germany \\ ${ }^{2}$ High Performance Computing Center Stuttgart, Nobelstr. 19, 70569 Stuttgart, Germany
}

(Received 13 October 2006 and in revised form 24 January 2007)

A central goal of flow control is to minimize the energy consumption in turbulent flows and nowadays the best results in terms of drag reduction are obtained with the addition of long-chain polymers. This has been found to be associated with increased anisotropy of turbulence in the near-wall region. Other drag reduction mechanisms are analysed in this respect and it is shown that close to the wall highly anisotropic states of turbulence are commonly found. These findings are supported by results of direct numerical simulations which display high drag reduction effects of over $30 \%$ when only a few points inside the viscous sublayer are forced towards high anisotropy.

\section{Introduction}

The question of how wall-bounded flows can be controlled with reasonable cost is of fundamental and practical importance since it is directly related to viscous drag and heat transfer processes. The most effective flow control with respect to minimizing the viscous drag is achieved by the addition of long-chain polymers. With a polymer concentration of only a few p.p.m., drag reductions (DR) of up to $80 \%$ can be achieved. Recently, it was shown that the drag reducing mechanism of polymers can be related to highly anisotropic states of near-wall turbulence (Jovanovic et al. 2006). In this work we analyse available data sets from direct numerical simulations (DNS) for other DR phenomena in this respect and show that these techniques have a common feature. This common mechanism of drag reduction is validated in a channel flow simulation with forced boundary conditions in the near-wall region. The identification of a common mechanism for drag reduction can serve as a design criterion in the development of new flow control techniques. In spite of existing mechanisms that lead to high DR, new developments are needed since each of the existing techniques has a limited application area, e.g. for environmental reasons flow additives cannot be used in outer flows.

\section{Anisotropy-invariant mapping of wall turbulence}

The level of anisotropy of turbulence can be quantified, following the analysis of Lumley \& Newman (1977), by introducing the anisotropy tensor

$$
a_{i j}=\frac{\overline{u_{i} u_{j}}}{q^{2}}-\frac{1}{3} \delta_{i j}
$$




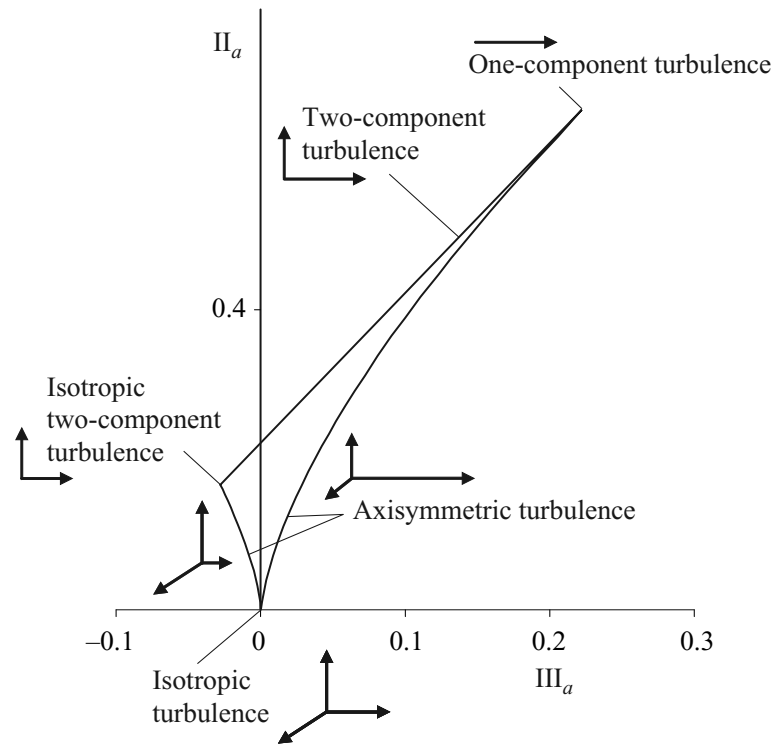

FIGURE 1. Anisotropy-invariant map showing the limiting states of turbulence. The arrows represent the turbulent fluctuations that correspond to each of the limiting states.

(where $q^{2}=\overline{u_{s} u_{s}}$ ) and its scalar invariants

$$
\begin{aligned}
\mathrm{II}_{a} & =a_{i j} a_{j i}, \\
\mathrm{III}_{a} & =a_{i j} a_{j k} a_{k i} .
\end{aligned}
$$

A plot of $\mathrm{II}_{a}$ versus $\mathrm{III}_{a}$ for axisymmetric turbulence,

$$
\mathrm{II}_{a}=\frac{3}{2}\left(\frac{4}{3}\left|\mathrm{III}_{a}\right|\right)^{2 / 3},
$$

and two-component turbulence,

$$
\mathrm{II}_{a}=\frac{2}{9}+2 \mathrm{III}_{a},
$$

defines the anisotropy-invariant map according to Lumley (1978). This plot, shown in figure 1 , bounds all physically realizable turbulence. The two curves shown represent axisymmetric turbulence. The right-hand curve corresponds to turbulence strained by axisymmetric expansion and the left-hand curve corresponds to straining by axisymmetric contraction. Two-component turbulence resides along the straight line. Such turbulence exists in the region of the viscous sublayer in wall-bounded turbulent flows. The limiting states of turbulence are located at the corner points on the right- and left-hand sides of the anisotropy-invariant map and correspond to onecomponent turbulence and isotropic two-component turbulence, respectively. In these limiting states, turbulence must satisfy the two-component limit and axisymmetry at large and small scales as discussed by Jovanović \& Hillerbrand (2005).

The influence of the Reynolds number on the anisotropy of turbulence in a plane channel flow is shown in figure 2, where $R e_{b}$ refers to the Reynolds number based on bulk velocity, channel half-width $\delta$ and kinematic viscosity $v$ of the working fluid and $R e_{\tau}=u_{\tau} \delta / \nu$ where $u_{\tau}$ is the wall shear velocity. Data that correspond to the region of the viscous sublayer, $0 \leqslant x_{2}^{+}<5$ (where $x_{2}^{+}$represents the normalized distance from the wall $\left.x_{2}^{+}=x_{2} u_{\tau} / v\right)$, lie along the two-component limit. Away from the near-wall region, $x_{2}^{+} \geqslant 8$, the trend in the data indicates a tendency towards the right boundary 

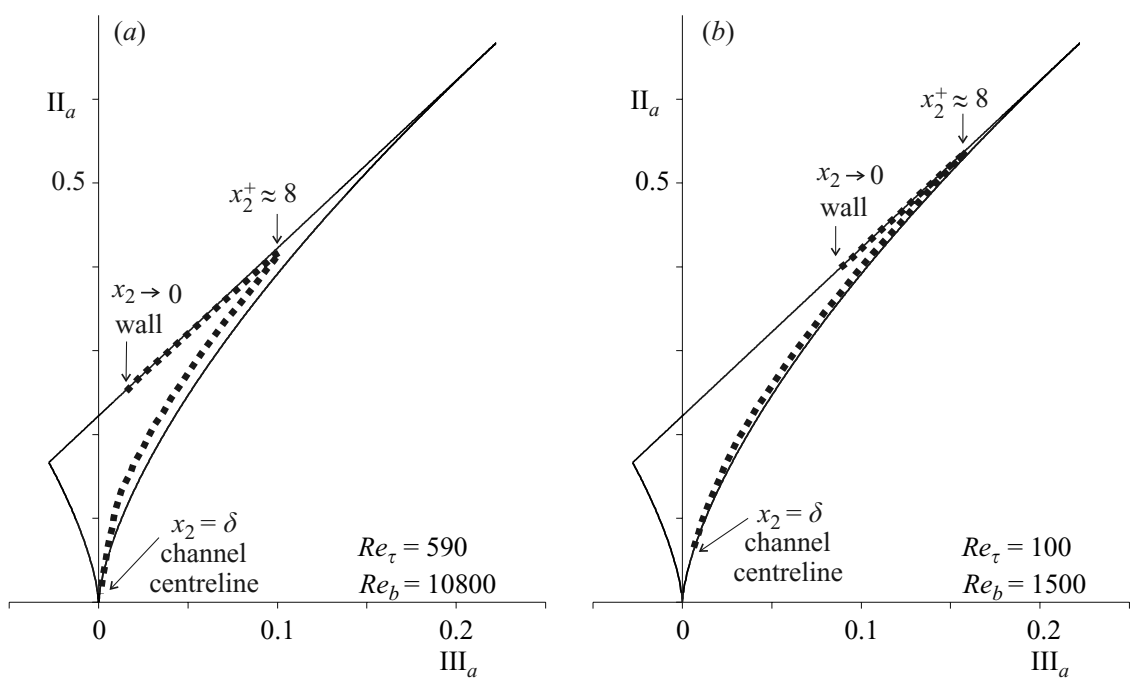

FiguRE 2. Trajectories of turbulent channel flow at (a) moderate (Moser, Kim \& Mansour 1999 ) and (b) low (Kuroda, Kasagi \& Hirata 1990) Reynolds numbers.

of the anisotropy-invariant map, which corresponds to the axisymmetric state with the streamwise intensity larger than the intensities in the other two directions. There is a noticeable trend in these data. As the Reynolds number decreases towards the critical value valid for transition from the laminar to the turbulent state, the anisotropy increases near the wall and data on the two-component limit tend towards the one-component state of turbulence. Away from the near-wall region, turbulence tends towards the axisymmetric state with the streamwise intensity larger than in the other two directions, $\overline{u_{1}^{2}}>\overline{u_{2}^{2}}=\overline{u_{3}^{2}}$. Since in axisymmetric turbulence there is no shear stress $-\overline{u_{1} u_{2}}$, it is natural to expect that in the limit when $R e \rightarrow(R e)_{\text {crit }}$ there will be no traditional mechanism of energy production, $P_{k}=-\bar{u}_{1} u_{2} \partial \bar{U}_{1} / \partial x_{2}$, which ensures self-maintenance of turbulence in wall-bounded flows.

\section{Polymer drag reduction}

It was reported previously (Jovanovic et al. 2006) that polymer drag reduction is accompanied by increasing anisotropy in the near-wall region. The experimental results obtained for measurements in the viscous sublayer were in close agreement with those obtained by analysing the DNS data of Dimitropoulos, Sureshkumar \& Beris (1998) in the anisotropy-invariant map, as shown in figure 3 . The trajectory for a channel flow without additives is plotted in $(a)$. The trajectories in $(b)$ and (c) correspond to turbulent flow fields at the same Reynolds number in which DR of $15 \%$ and $44 \%$ was achieved by the addition of polymers. It can clearly be seen that the anisotropy of near-wall turbulence $\left(x_{2} \rightarrow 0\right)$ is shifted to higher values as drag reduction increases. This trend of increased anisotropy corresponds to the one observed in turbulent channel flows without additives for decreasing Reynolds numbers (see figure 2).

A conceptual sketch of the interaction between polymer and turbulence (Jovanovic et al. 2006) is given in figure 4. Under very special circumstances when the polymer concentration and its relaxation time are appropriately matched to the properties of the turbulence, turbulence in the near-wall region forces rolled-up chains of polymer 

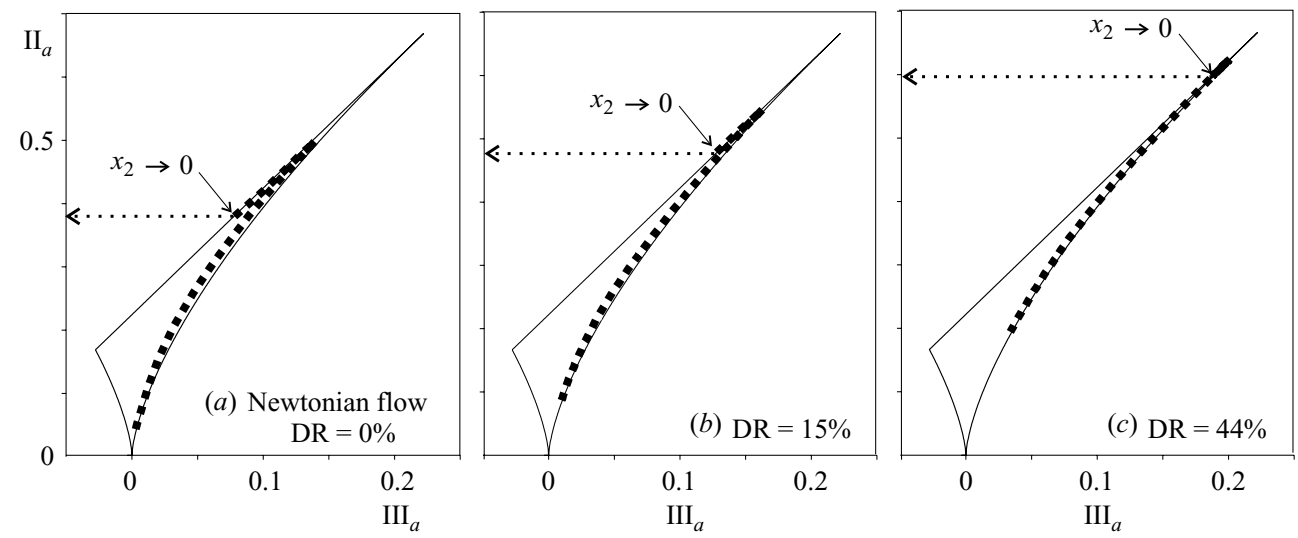

FIGURE 3. Anisotropy-invariant mapping of turbulence in a fully developed channel flow with DR from direct numerical simulations of Dimitropoulos et al. (1998) at $R e_{b}=1840$. The trend in the data at the wall $\left(x_{2} \rightarrow 0\right)$ demonstrates that DR increases as turbulence approaches the one-component limit.
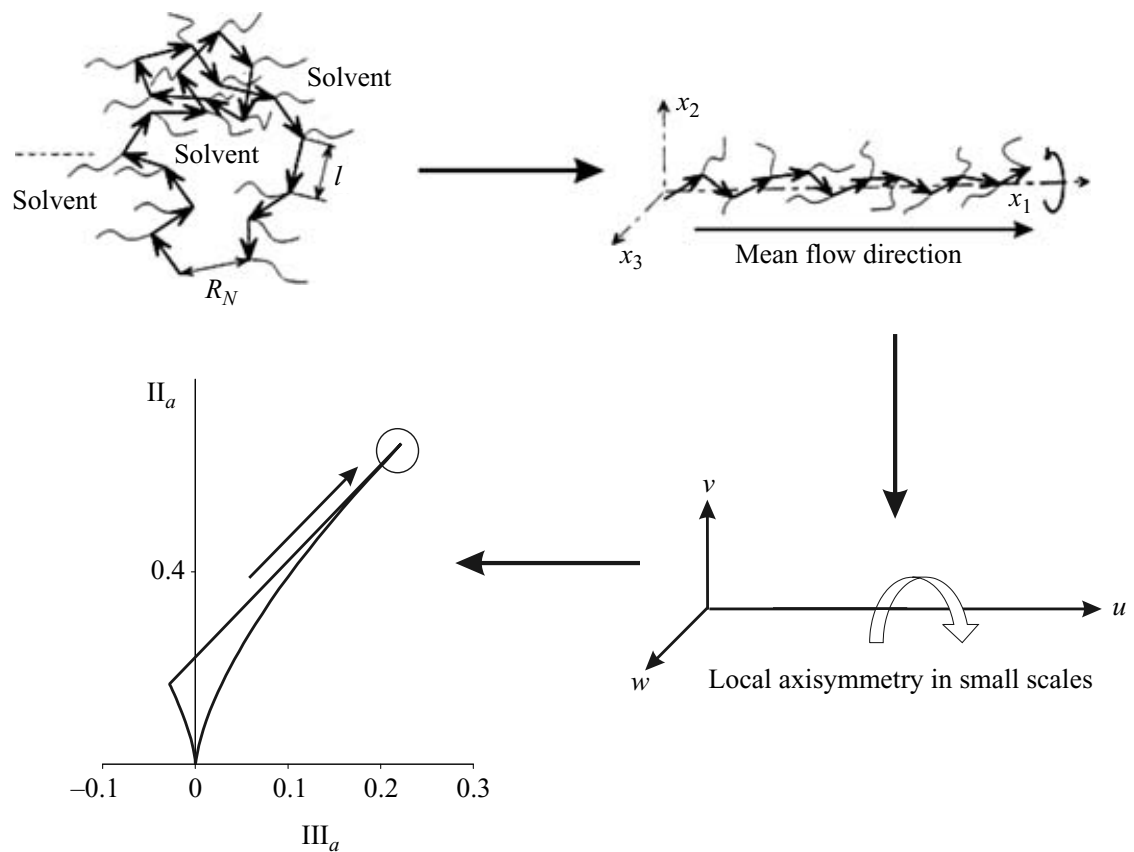

FIGURE 4. Conceptual sketch for the interaction of polymer and turbulence. When the polymer is stretched in the mean flow direction, it forces the smallest scales of turbulence to restructure. This change is reflected in the anisotropy-invariant map as a movement towards the one-component limit for the near-wall turbulence since this is the stage where two-component turbulence and axisymmetric turbulence can be achieved simultaneously (in pipe and channel flows).

partially to unroll and stretch in the mean flow direction. In the most extreme case, polymer chains form a filament structure with a length-scale arrangement which is almost axisymmetric around the axis aligned with the mean flow. This process forces turbulence at small scales to restructure and satisfy constraints of local axisymmetry. 

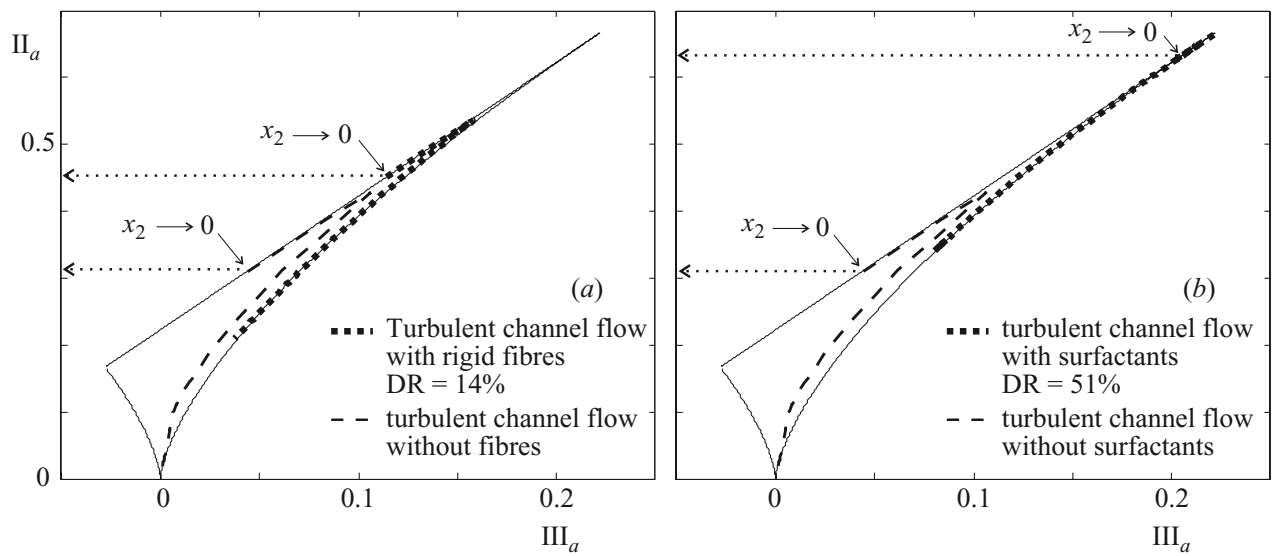

FIGURE 5. Anisotropy-invariant mapping of a turbulent channel flow with $(a)$ rigid fibres and (b) surfactant additives in comparison with a fully developed turbulent channel flow without additives at the same Reynolds number $R e_{b} \simeq 6000$.

In the near-wall region where turbulence is two-component and therefore located on the upper line of the anisotropy-invariant map, the axisymmetric state with the dominant streamwise component on the axis of rotational invariance can only be reached by approaching the one-component limit $\left(\mathrm{II}_{\mathrm{a}}=2 / 3\right)$ where axisymmetry and two-componentality are simultaneously fulfilled.

The above-mentioned conceptual mechanism is based on the assumption that the interaction between polymer and turbulence takes place in the dissipation range of the spectrum so that only the smallest turbulence scales interact with the polymer. This interaction level at the Kolmogorov scale was verified in Jovanovic et al. by the theoretical prediction of optimum polymer concentration and optimum molecular weight of the polymer to obtain maximum DR.

\section{Is there a general mechanism of drag reduction?}

The analysis of polymer DR has shown that high DR in wall-bounded flows can be obtained by forcing the velocity fluctuations near the wall towards axisymmetry. It is of great interest for the design of passive and active flow control techniques to investigate whether the same trend can be found in other drag-reduced flows. Therefore, the DNS databases of different drag-reduced flows are analysed in this respect.

Fibres and surfactants are also additives that can lead to high DR. DNS results are available through the work of Paschkewitz et al. (2004) and Kawaguchi et al. (2005). The analysis of their results in the anisotropy-invariant map is shown in figure 5. The trajectories are plotted in comparison with that for a turbulent channel flow without additives at the same Reynolds number. As for polymer DR, a substantial increase in the anisotropy in the near-wall region is found for the drag-reduced flows.

In passive flow control, surface modifications known as riblets have been extensively investigated. The highest DR was obtained with a geometry of very thin riblets suggested by Bechert et al. (2000). In Lammers, Jovanovic \& Durst (2006) the latticeBoltzmann method was used to simulate a channel flow with this kind of wall configuration. In contrast to the flows with additives, surface structures introduce 

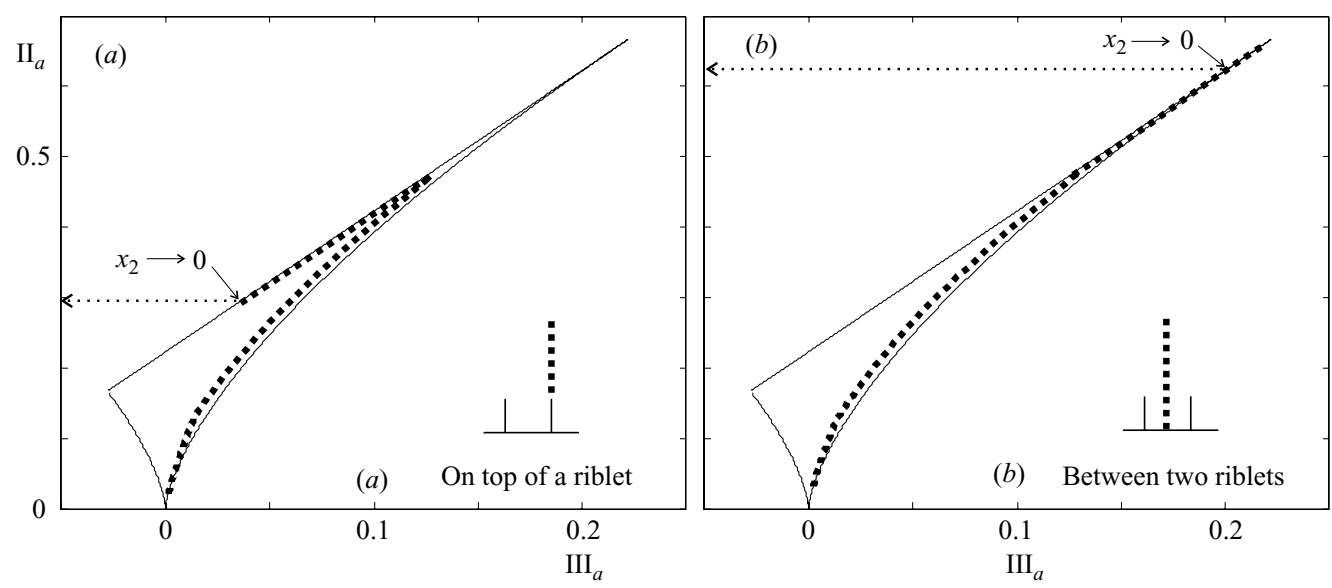

FIGURE 6. Anisotropy-invariant mapping of the flow $(a)$ on top of a riblet and $(b)$ between two riblets at $R e_{b}=2800$.

variances in the spanwise direction so that the flow field can be analysed along different cross-sectional areas (aligned in the flow direction and perpendicular to the walls). The trajectories for the flow above a riblet and for the flow between two riblets are presented in figure 6 . The trajectory on top of the riblet resembles very closely that of a plain turbulent channel flow at the same Reynolds number whereas the trajectory between two riblets shows a significant increase in the anisotropy near the wall. The resulting DR is significantly lower than that obtained with long-chain polymers since the one-component limit can only be approached locally and not along the entire surface of the wall.

DR is also observed for highly accelerated and supersonic flows. Spalart (1986) and Foysi, Sarkar \& Friedrich (2004) provided DNS data sets for these flows. Highly accelerated flows are known to relaminarize if the acceleration parameter $K$ :

$$
K=\frac{v}{U_{\infty}^{2}} \frac{\mathrm{d} U_{\infty}}{\mathrm{d} x},
$$

exceeds a critical value in the order of $K_{\text {crit }} \geqslant 3.7 \times 10^{-6}$ (Spalart 1986). The trajectories of highly accelerated boundary layer flows approaching the critical value of $K$ are shown in figure 7. With increasing acceleration parameter a shift in the wall points $\left(x_{2} \rightarrow 0\right)$ towards the one-component limit is observed.

In supersonic flows, the skin friction coefficient normalized with the corresponding value valid for subsonic flow $c_{f} / c_{f_{M a=0}}$ decreases with increasing Mach number; $M a$, as pointed out by Hinze (1975). From the simulations of Foysi et al. (2004), DNS data sets are available for Mach numbers $0.3,1.5,3.0$ and 3.5. The analysis of these data sets is shown in figure 8 . It can clearly be seen that the marked wall points assume more anisotropic states as the Mach number is increased.

The analysis presented of different DR mechanisms in the anisotropy-invariant map suggests that a common feature exists. In order to achieve high DR, near-wall turbulence is shifted in the direction of the one-component limit at the wall, which is formally equivalent to forcing the small scales of turbulence to fulfil conditions of local axisymmetry. 

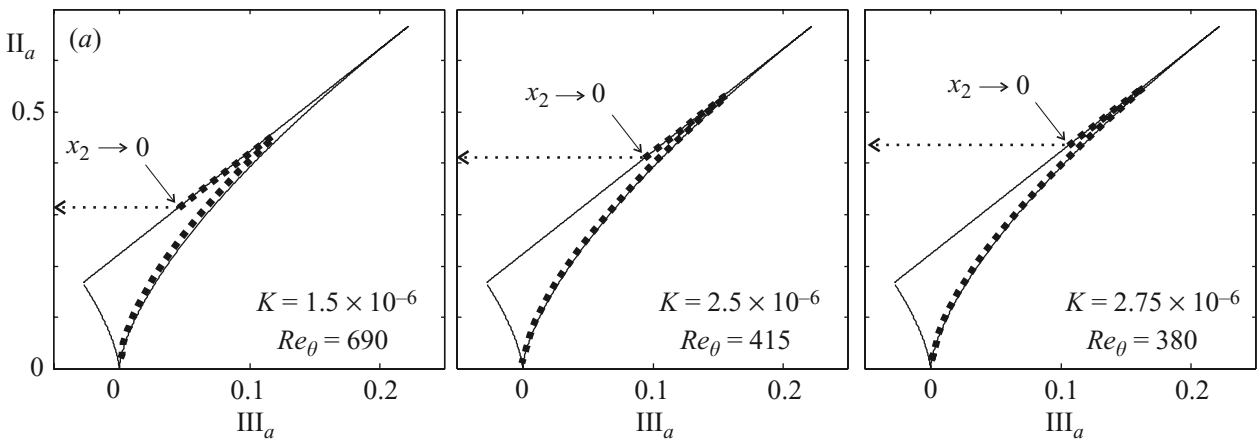

FIGURE 7. Anisotropy-invariant mapping of highly accelerated boundary layers at different acceleration parameters. The severe acceleration results in a decrease of the Reynolds number $R e_{\theta}$ based on the momentum thickness.
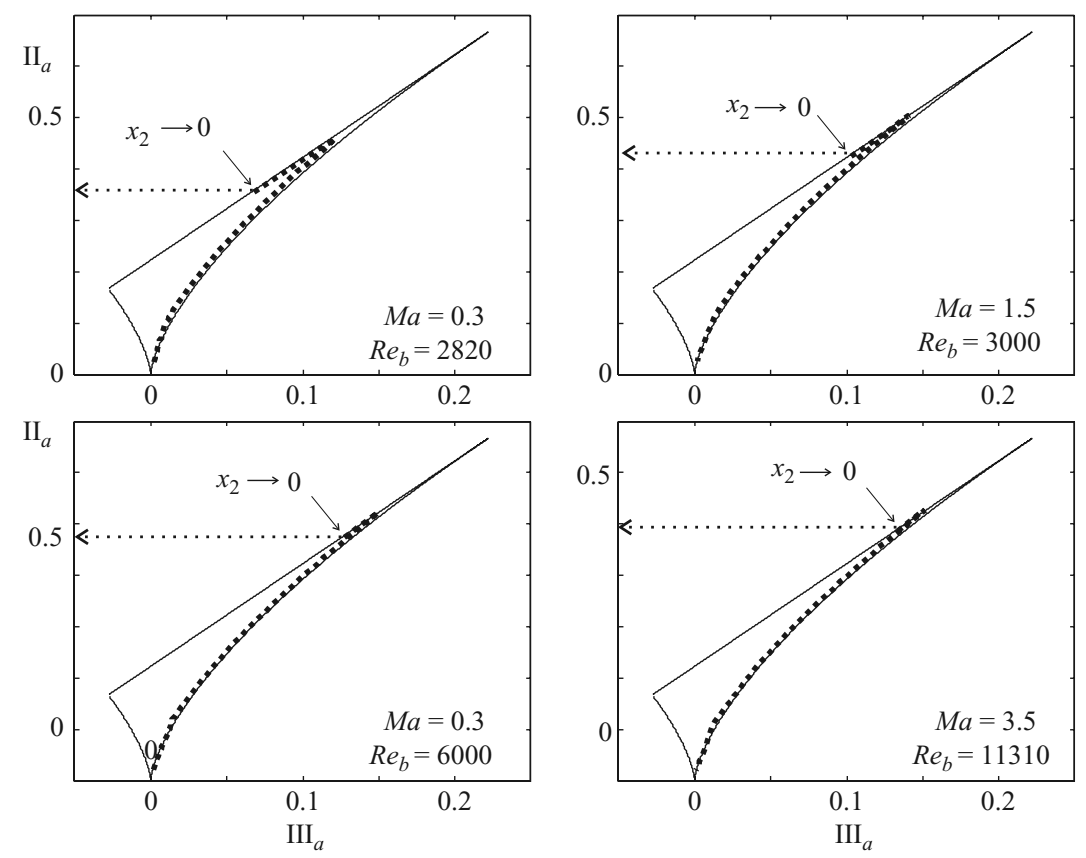

FIGURE 8. Anisotropy-invariant mapping of supersonic flows. As the Mach number (and also the bulk Reynolds number) is increased, the wall points show a shift towards the one-component limit.

\section{Channel flow with forced boundary conditions}

To demonstrate the effect of increasing anisotropy in the near-wall region on drag reduction, direct numerical simulations of a turbulent channel flow with forced boundary conditions were carried out. A set of five simulations was performed using a fourth-order finite-volume method on a staggered grid as described in Verstappen \& Veldman (2003). In one simulation at $R e_{\tau}=\delta u_{\tau} / \nu=180$ no forcing was applied to the flow. For the other simulations the flow rate and thus also the bulk Reynolds number $R e_{b}$ were kept constant but at the first, first two, first three and first four points (located at $x_{2} / H=0.0004,0.012,0.020,0.028$ which corresponds to $x_{2}^{+}=0.7,2.2,3.6,5.1$ 

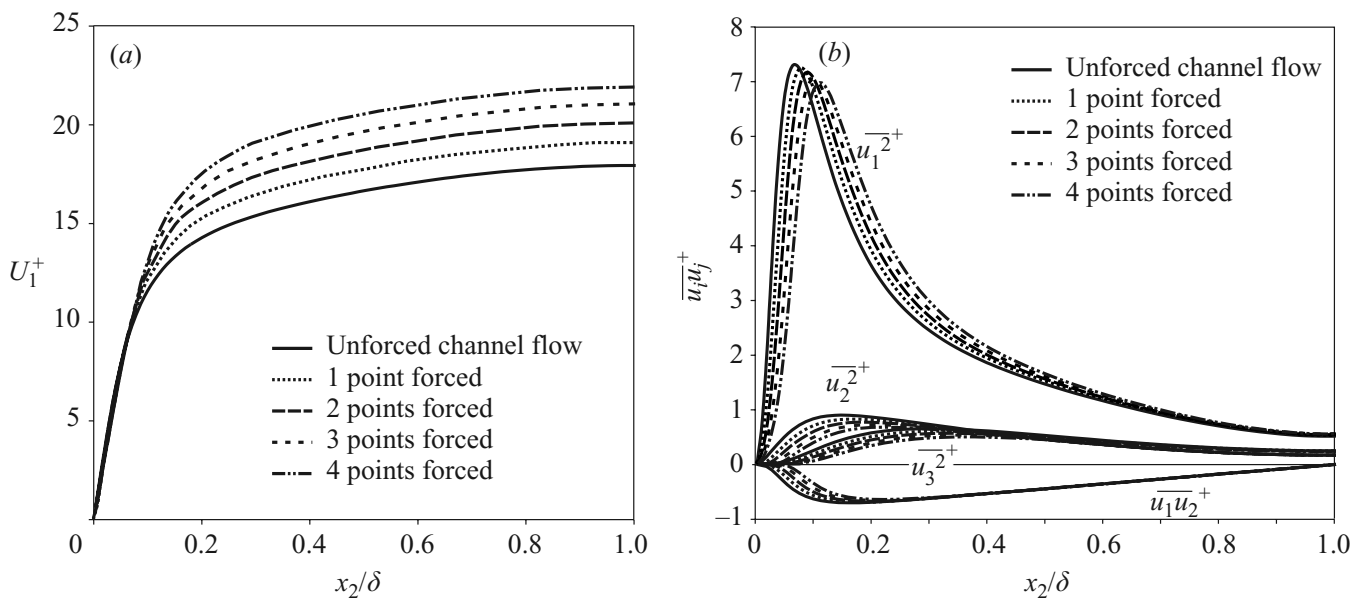

Figure 9. (a) Mean flow and (b) Reynolds stresses in a virtual channel flow at $\operatorname{Re}_{b}=2800$ where an increasing number of points in the viscous sublayer are forced towards an axisymmetric state. The quantities are normalized with the corresponding wall shear velocity $u_{\tau}$ of each simulation.

in the unforced channel flow) the boundary conditions were modified in such a way as to force near-wall turbulence to tend towards an axisymmetric state by imposing that spanwise velocity fluctuations $\left(u_{3}\right)$ follow the normal fluctuations $\left(u_{2}\right)$.

The applied forcing yielded decreasing values of $R e_{\tau}$. The resulting mean flow velocity profile and the Reynolds stresses (normalized with the respective $u_{\tau}$ of each flow case) are shown in figure 9. It can be seen that $U_{1}^{+}$increases significantly as the forcing is applied to more points inside the viscous sublayer, which is equivalent to a decrease in $u_{\tau}$ and thus a reduced wall shear stress $\tau_{w}$. Since $\tau_{w}$ and $R e_{\tau}$ are related by

$$
\tau_{w}=\rho\left(\frac{\nu R e_{\tau}}{\delta}\right)^{2}
$$

and since the fluid properties $\rho, v$ and the channel height $H=2 \delta$ were kept constant, the drag reduction DR obtained is given by

$$
\mathrm{DR}=1-\left(\frac{R e_{\tau}}{\left(R e_{\tau}\right)_{180}}\right)^{2},
$$

resulting in $\mathrm{DR}=12 \%, 20 \%, 27 \%$ and $32 \%$ respectively.

The resulting trajectories in the anisotropy-invariant map are all very similar, with the wall point located at the one-component limit (as it was forced to). Figure 10(a) shows the trajectory for the case where four points in the viscous sublayer were forced towards an axisymmetric state which resulted in DR $=32 \%$. In figure $10(b)$, an enlarged region of the anisotropy-invariant map is shown in which the trajectories for the five simulations are plotted. The part of the trajectory shown corresponds to the region of $x_{2}^{+} \approx 40$ for the unforced channel flow. There is a clear trend in the data towards the right branch of the map, which represents the axisymmetric state with the dominant fluctuations on the axis of invariance. This trend implies that the forcing inside the viscous sublayer, $x_{2}^{+} \leqslant 5$, has introduced a general shift towards the right branch of the anisotropy-invariant map. 


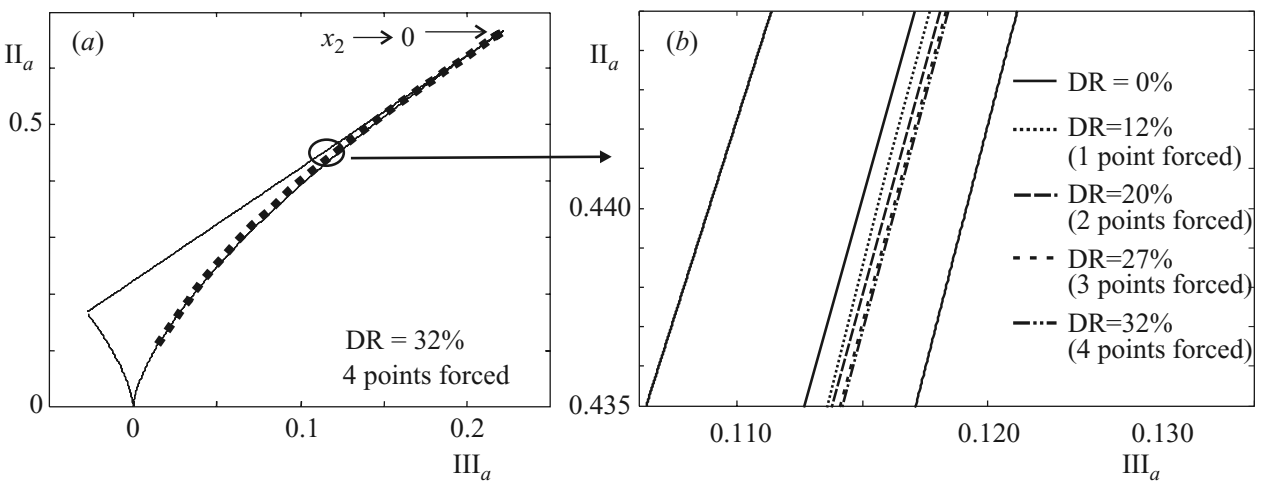

FiguRE 10. (a) Anisotropy-invariant mapping of turbulent channel flow at $R e_{b}=2800$ where four points in the viscous sublayer are forced towards an axisymmetric state. ( $b$ ) An enlargement of (a) showing part of the trajectories in the anisotropy-invariant map for simulations at the same bulk Reynolds number but with different boundary conditions.

\section{Conclusions}

The analysis of DR in the terminology of anisotropy invariants provides a suggestion for how turbulence needs to be modified in order to achieve energy savings. In all of the examples of drag-reduced flows discussed, it is seen that the anisotropy of turbulence increases towards the one-component limit in the near-wall region. The same trend is observed if the Reynolds number in turbulent flows is reduced. The one-component limit is the only point in the anisotropy-invariant map where two-componentality (which has to hold at the wall) and axisymmetry with the dominant fluctuation on the axis of rotational invariance can simultaneously be fulfilled.

The conclusion to be drawn from the analysis for the design of active and passive flow control techniques presented is that near-wall fluctuations need to be predominantly one-component in the mean flow direction in order to achieve high values of DR and energy savings. The direct numerical simulations presented demonstrate that in this way very effective flow control can be exerted in which little effort is needed to achieve significant energy savings.

This work is sponsored by the Deutsche Forschungsgemeinschaft under grant number Jo240/5-1. The computations were carried out on a NEC SX at the HLRS. The authors gratefully acknowledge this support.

\section{REFERENCES}

Bechert, D. W., Bruse, M., Hage, W. \& Meyer, R. 2000 Fluid mechanics of biological surfaces and their technological application. Naturwissenschaften 87, 157-171.

Dimitropoulos, C. R., Sureshrumar, R. \& Beris, A. N. 1998 Direct numerical simulation of viscoelastic turbulent channel exhibiting drag reduction: effect of the variation of rheological parameters. J. Non-Newtonian Fluid Mech. 79, 443-468.

Foysi, G., SARKar, S. \& Friedrich, R. 2004 Compressibility effects and turbulence scaling in supersonic flows. J. Fluid Mech. 509, 207-216.

Hinze, J. O. 1975 Turbulence, 2nd Edn. McGraw-Hill.

Jovanović, J. \& Hillerbrand, R. 2005 On peculiar property of the velocity fluctuations in wallbounded flows. J. Thermal Sci. 9, 3-12. 
Jovanović, J., Pashtrapanska, M., Frohnapfel, B., Durst, F., Koskinen, J. \& Koskinen, K. 2006 On the mechanism responsible for turbulent drag reduction by dilute addition of high polymers: theory, experiments, simulations, and predictions. Trans. ASME: J. Fluids Engng 128, $118-130$.

Kawaguchi, Y., Li, F. C., Bo, Y. \& Hishida, K. 2005 Experimental and numerical investigation of the mechanism of drag reduction by surfactant additives and heat transfer. Proc. 6th Symp. Smart Control of Turbulence, Tokyo. http://www.nmri.go.jp/turbulence/ PDF/symposium/FY2004/kawaguchi.pdf

Kuroda, A., Kasagi, N. \& Hirata, M. 1990 A direct numerical simulation of the fully developed turbulent channel flow. Proc. Intl Symp. Comp. Fluid Dynamics, Nagoya (ed. M. Yasuhara, H. Daiguji, K. Oshima), pp. 1174-1179. JSCFD.

Lammers, P., Jovanović, J. \& Durst, F. 2006 Numerical experiments on wall turbulence at low Reynolds numbers. J. Thermal Sci. 10, 33-62.

Lumley, J. L. 1978 Computational modeling of turbulent flows. Adv. Appl. Mech. 18, 123-176.

Lumley, J. L. \& Newman, G. 1977 The return to isotropy of homogeneous turbulence. J. Fluid Mech. 82, 161-178.

Moser, R. D., Kim, J. \& Mansour, N. N. 1999 DNS of turbulent channel flow up to $R e_{\tau}=590$. Phys. Fluids 11, 943-945.

Paschkewitz, J. S., Dubief, Y., Dimitropoulos, C. D., Shaqfeh, E. S. \& Moin, P. 2004 Numerical simulation of turbulent drag reduction using rigid fibers. J. Fluid Mech. 518, 281-317.

Spalart, P. R. 1986 Numerical study of sink-flow boundary layer. J. Fluid Mech. 172, 307-328.

Verstappen, R. W. C. P. \& Veldman, A. E. P. 2003 Symmetry-preserving discretization of turbulent flow. J. Comp. Phys. 187, 343-368. 